\title{
An Inexact Two-Stage Stochastic Programming Model for Sustainable Utilization of Water Resource in Dalian City
}

\author{
Binglong Wang ${ }^{1}$, Yanpeng Cai ${ }^{1,2}$, Linyu $\mathrm{Xu}^{1}$ and Wencong Yue ${ }^{1}$ \\ ${ }^{1}$ State Key Laboratory of Water Environment Simulation, School of Environment, \\ Beijing Normal University, Beijing, China \\ ${ }^{2}$ State Key Laboratory of Hydraulics and Mountain River Engineering, Sichuan \\ University, Chengdu, China \\ bnuwbl@163.com,yanpeng.cai@bnu.edu.cn
}

\begin{abstract}
An inexact two-stage stochastic programing model was applied to sustainable utilization of water resource under uncertainty in Dalian city. The developed model, integrated the two-stage stochastic programing and inexact optimization, could deal with uncertain problems expressed as probability distributions and discrete intervals. After formulating the model, a hypothetical case based on the comprehensive planning of sustainable utilization of water resource in Dalian city was employed for demonstrating its application in the three different planning year, which was 2015, 2020, 2030. The optimal allocation of water resource with maximized system benefit among different users had been obtained. The result indicated that different water allocation schemes and water deficits with varying net system benefits would be hold in response to different streamflow levels. In a word, The ITSP model provided significant decision support that adjusted to the existing schemes of water allocation within a complex water resource system under uncertainty for water managers.
\end{abstract}

Keywords: Sustainable utilization, Two-stage, Optimization, Uncertainty

\section{Introduction}

Over several decades, the main challenges in the domain of water resources management are resolving the varying levels of water shortage and deterioration of water quality caused by growing population, developing economic[1]. Worldwide, it is estimated that 768 million people remain without access to improving source of water and the number of people whose rights to water are not satisfied could reach up to 3.5 billion [2]. Besides, the uneven distribution of water resources in time and space scale exacerbated the water shortage problems[3]. To address these difficulties, effective management with environmental benefits and sound economic are expected to optimize the allocation of water resource.

Many stochastic optimization theories have been put forward to tackle with uncertain problems in the management of water resource system [4-9]. Two-stage stochastic programming (TSP) was considered as one of efficient methods that could deal with randomness. Wang and Adams (1986) proposed a framework of two-stage programming in response to the optimization of reservoir operation [10]. Edirisinghe and Ziemba (1994) proposed lower and upper bounds within two-stage stochastic linear programming (TSLP) through limited moment information[11]. Barik et al. (2014) developed a two-stage stochastic linear programming model considering some parameters as multi-choice parameters and others as exponential random variables[12].

However, there are some limitations in its application though TSP provided reasonable solutions for solving water allocation problems [13]. For example, TSP can handle uncertain problems presented as probability density functions (PDFs) and account for 
economic "penalties" with recourse against infeasibility, but it is hard to deal with uncertain coefficients in constraints' left side. In addition, as the data for producing PDFs are always insufficient, probabilistic specifications for parameters under uncertainty could not be very realistic in many practical problems[14].So how to define the PDFs and degree of confidence level that closer to the objective world will need to be further studied [15]. To deal with these uncertain problems with the TSP method, the inexact twostage stochastic programming (ITSP) model has already attracted widespread attentions over the past decades [16-19]. Huang and Loucks (2000) proposed the ITSP framework and applied the model to uncertain problem of water resources management[16]. Luo et al. (2003) developed an inexact two-stage stochastic nonlinear programming (ITSNP) model through water trading with great uncertainties[20]. The ITSP method, integrated the TSP and interval parameter programming (IPP), has two obvious characteristics: a) could deal with uncertainties expressed as probability distributions and discrete intervals, b) when the uncertain problems are resolved and random variables are known, the second stage decision can be undertaken to minimize "recourse cost" that may generate because of infeasibility[21].

\section{Modeling Formulation}

As the total water available has the feature of randomness, the problems that water managers concerned could be specialized by a two-stage stochastic programming (TSP) model[16]:

$=\sum_{i=1}^{m} N B_{i} T_{i}-\mathrm{E}\left[\sum_{i=1}^{m} C_{i} D_{i Q}\right]$,

s.t $Q \geq \sum_{i=1}^{m}\left(T_{i}-D_{i Q}\right)$,

$T_{\text {imax }} \geq T_{i} \geq D_{i Q} \geq 0, \quad i=1,2, \ldots, m$

where:

$f$ Net system benefit

$i$ Water user

m Total number of water users

$T_{i}$ Allocation target for water resource promised to user i

$N B_{i}$ Net benefit to user i per unit of water allocated

$C_{i}$ Recourse cost to user i per unit of water allocation not delivered $\left(C_{i} \geq N B_{i}\right)$

$\mathrm{D}_{i Q}$ Deficits by which water-allocation target $T_{i}$ is not met when the streamflow is $\mathrm{Q}$

$\mathrm{E}(\mathrm{x})$ Expectation of a random variable

Q Total available water with randomness

$T_{\text {imax }}$ Target of maximum allowable water-allocation for user $\mathrm{i}$

In order to solve the problems with the linear programming (LP) method, the distribution of random variable $Q$ should be expressed by a discrete function approximatively. Let $\mathrm{Q}$ take the value $\mathrm{q}_{\mathrm{h}}$ with probability $\mathrm{p}_{\mathrm{h}}(\mathrm{h}=1,2, \ldots, \mathrm{n})$, then we get:

$$
\mathrm{E}\left[\sum_{i=1}^{m} C_{i} D_{i Q}\right]=\sum_{i=1}^{m} C_{i} \times \sum_{h=1}^{n} p_{h} D_{i h}
$$

Therefore, the model (1) could be transformed into:

$$
\max f=\sum_{i=1}^{m} N B_{i} T_{i}-\sum_{i=1}^{m} C_{i} \sum_{h=1}^{n} p_{h} D_{i h}
$$




$$
\begin{aligned}
& \text { s.t } q_{h} \geq \sum_{i=1}^{m}\left(T_{i}-D_{i h}\right), \quad \forall i, h \\
& T_{\text {imax }} \geq T_{i} \geq D_{\text {ih }} \geq 0, \quad \forall i, h
\end{aligned}
$$

where $D_{\text {ih }}$ is the water deficit by which fixed allowable water-allocation target $T_{i}$ is not satisfied when the streamflow is $\mathrm{q}_{\mathrm{h}}$ with probability $\mathrm{p}_{\mathrm{h}}$. The above model can reflect uncertainty in the process of water availability. But the related economic data and waterallocation targets are rarely available as reliable probability distributions or deterministic values. As a consequence, interval variables and parameters are integrated into the TSP framework to handle uncertain problems by introducing $\mathrm{T}_{\mathrm{i}}^{ \pm}, \mathrm{NB}_{\mathrm{i}}^{ \pm}$, and $\mathrm{C}_{\mathrm{i}}^{ \pm}$into the analysis and calculating process. So it lead to a hybrid inexact two-stage stochastic programming model $[16,22]$ generated as follows:

$$
\begin{aligned}
& \max f^{ \pm}=\sum_{i=1}^{m} N B_{i}{ }^{ \pm} T_{i}{ }^{ \pm}-\sum_{i=1}^{m} \sum_{h=1}^{n} p_{h} C_{i}^{ \pm} D_{i h}^{ \pm} \\
& \text {s.t } \quad q_{h}^{ \pm} \geq \sum_{i=1}^{m}\left(T_{i}^{ \pm}-D_{i h}^{ \pm}\right), \quad \forall h \\
& T_{\text {imax }}^{ \pm} \geq T_{i}^{ \pm} \geq D_{i h}^{ \pm} \geq 0, \quad \forall i, h
\end{aligned}
$$

where $\mathrm{T}_{\mathrm{i}}^{ \pm}, \mathrm{T}_{\mathrm{imax}}{ }^{ \pm}, \mathrm{NB}_{\mathrm{i}}{ }^{ \pm}, \mathrm{p}_{\mathrm{h}}{ }^{ \pm}, \mathrm{C}_{\mathrm{i}}^{ \pm}$and $\mathrm{D}_{\mathrm{ih}}{ }^{ \pm}$are interval variables or parameters. As three different planning year are considered, the model (4) would be reformulated as:

$$
\begin{aligned}
& \max f^{ \pm}=\sum_{i=1}^{m} N B_{i t}{ }^{ \pm} T_{i t}{ }^{ \pm}-\sum_{i=1}^{m} \sum_{h=1}^{n} p_{h} C_{i t}^{ \pm} D_{i h t}^{ \pm} \\
& \text {s.t } \quad q_{h}^{ \pm} \geq \sum_{i=1}^{m}\left(T_{i t}^{ \pm}-D_{i h t}^{ \pm}\right), \quad \forall h, t \\
& T_{i t \text { max }}^{ \pm} \geq T_{i t}^{ \pm} \geq D_{i h t}^{ \pm} \geq 0, \quad \forall i, h, t
\end{aligned}
$$

where $\mathrm{T}_{\mathrm{i}}^{ \pm}, \mathrm{T}_{\mathrm{imax}}{ }^{ \pm}, \mathrm{NB}_{\mathrm{i}}{ }^{ \pm}, \mathrm{p}_{\mathrm{h}}{ }^{ \pm}, \mathrm{C}_{\mathrm{i}}^{ \pm}$and $\mathrm{D}_{\mathrm{ih}}{ }^{ \pm}$are interval variables or parameters. As three different planning year are considered, the model (4) would be reformulated as:where $t$ is the planning year.

Let $\mathrm{T}_{\mathrm{it}}{ }^{+}$and $\mathrm{T}_{\mathrm{it}}{ }^{-}$be the upper and lower bound of $\mathrm{T}_{\mathrm{it}}{ }^{ \pm}$, and then we have $\mathrm{T}_{\mathrm{it}}{ }^{ \pm}=\left[\mathrm{T}_{\mathrm{it}}{ }^{-}, \mathrm{T}_{\mathrm{it}}{ }^{+}\right]$. While the values of $\mathrm{T}_{\mathrm{it}}{ }^{ \pm}$are known, the model (5) could be converted to two sets of submodels, which are corresponded to the lower and upper bound of the objective function value[23].Based on interactive algorithm, the transformation process, which is quite different from traditional best/worst case analysis, could provide a solution of stable intervals for both the decision variable and desired objective function. It is clearly interpreted for how to generate decision alternatives. The concrete steps of transformation process are as followed.

Let $\mathrm{T}_{\mathrm{it}}{ }^{ \pm}$have a deterministic value of $\mathrm{T}_{\mathrm{it}}{ }^{-}+\Delta \mathrm{T}_{\mathrm{it}} \mathrm{y}_{\mathrm{it}}$, where $\Delta \mathrm{T}_{\mathrm{it}}=\mathrm{T}_{\mathrm{it}}{ }^{+}-\mathrm{T}_{\mathrm{it}}{ }^{-}$and $0 \leq \mathrm{y}_{\mathrm{it}} \leq 1$. Model (5) can be rewritten as:

$$
\begin{aligned}
& \max f^{ \pm}=\sum_{i=1}^{m} N B_{i t}{ }^{ \pm}\left(T_{i t}^{-}+\Delta T_{i t} y_{i t}\right)-\sum_{i=1}^{m} \sum_{h=1}^{n} p_{h} C_{i t}^{ \pm} D_{i h t}^{ \pm} \\
& \text {s.t } q_{h}^{ \pm} \geq \sum_{i=1}^{m}\left(T_{i t}^{-}+\Delta T_{i t} y_{i t}-D_{i h t}^{ \pm}\right), \quad \forall h, t
\end{aligned}
$$




$$
\begin{gathered}
T_{i t m a x}^{ \pm} \geq T_{i t}^{-}+\Delta T_{i t} y_{i t} \geq D_{i h t}^{ \pm} \geq 0, \quad \forall i, h, t \\
0 \leq y_{i t} \leq 1, \quad \forall \mathrm{i}, \mathrm{t}
\end{gathered}
$$

To put all of decision variables on the left-hand side of constraints, the model (6b) and (6c) could be rewritten as follows:

$$
\begin{aligned}
& \sum_{i=1}^{m}\left(\Delta T_{i t} y_{i t}-D_{i h t^{ \pm}}\right) \leq q_{h}^{ \pm}-\sum_{i=1}^{m} T_{i t}^{-}, \quad \forall h, t \\
& \Delta T_{i t} y_{i t} \leq T_{i t m a x}^{ \pm}-T_{i t}^{-}, \quad \forall i, t \\
& D_{i h t}^{ \pm}-\Delta T_{i t} y_{i t} \leq T_{i t}^{-}, \quad \forall i, h, t \\
& D_{i h t}^{ \pm} \geq 0, \quad \forall i, h, t
\end{aligned}
$$

The upper bound of the corresponding objective function as follows:

$$
f^{+}=\sum_{i=1}^{m} N B_{i t}{ }^{+}\left(T_{i t}^{-}+\Delta T_{i t} y_{i t}\right)-\sum_{i=1}^{m} \sum_{h=1}^{n} p_{h} C_{i t}^{ \pm} D_{i h t}^{ \pm}
$$

Based on (7) and (8), high benefit are obtained provided that water demand could be satisfied when $\mathrm{T}_{\text {it }}{ }^{t}$ reach their upper bounds, and meanwhile they may have to pay high penalties if the promised water is not allocated to water users as scheduled. Conversely, when $\mathrm{T}_{\text {it }}{ }^{ \pm}$approach the lower bounds, lower benefit could be obtained with lower risk if against the promised target. So it is often hard to identify whether $\mathrm{T}_{\text {it }}{ }^{+}$or $\mathrm{T}_{\text {it }}{ }^{-}$would be corresponded to the upper bound of the desired objective function.

Therefore, if $\mathrm{T}_{\mathrm{it}}{ }^{ \pm}$are regarded as input parameters under uncertainty, the methods to solve problems of the inexact linear stochastic programming could be not directly used. In this paper, the optimized set of target values would be gained by introducing decision variable $y_{\text {it }}$ into model (6). Thus the optimized set will be corresponded to the uppermost bound of net benefit When uncertainty exist in the constraints' right-hand side, the submodel that correspond to $\mathrm{f}^{+}$should be related to upper bound of the right-hand side. Thus, the sub-models for $\mathrm{f}^{+}$are as followed:

$$
\begin{aligned}
& \max f_{t}^{+}=\sum_{i=1}^{m} N B_{i t}{ }^{+}\left(T_{i t}^{-}+\Delta T_{i t} y_{i t}\right)-\sum_{i=1}^{m} \sum_{h=1}^{n} p_{h} C_{i t}^{-} D_{i h t}^{-} \\
& \text {s.t. } \quad \sum_{i=1}^{m}\left(\Delta T_{i t} y_{i t}-D_{i h t}{ }^{-}\right) \leq q_{h}{ }^{+}-\sum_{i=1}^{m} T_{i t}^{-}, \quad \forall h, t \\
& \Delta T_{i t} y_{i t} \leq T_{i t m a x}^{+}-T_{i t}^{-}, \quad \forall h, t \\
& D_{\text {iht }}^{-}-\Delta T_{i t} y_{i t} \leq T_{i t}^{-}, \quad \forall i, h, t \\
& D_{\text {iht }}^{-} \geq 0, \quad \forall i, h, t \\
& 0 \leq y_{i t} \leq 1, \quad \forall i, t
\end{aligned}
$$

Solution of model (9) provides the highest system benefit, which considered uncertainty in the inputs of allowable water-allocation targets. Let $\mathrm{D}_{\text {ihtopt }}$ and $\mathrm{y}_{\text {itopt }}$ be solutions of the model (9). So the optimized targets of allowable water-allocation are as 
followed:

$$
T_{i t o p t}^{ \pm}=T_{i t}^{-}+\Delta T_{i t} y_{i t}, \quad \forall h, t
$$

Thus, we have the sub modes for $\mathrm{f}$ as follows:

$$
\begin{aligned}
& \max f_{t}^{-}=\sum_{i=1}^{m} N B_{i t}{ }^{-}\left(T_{i t}^{-}+\Delta T_{i t} y_{\text {itopt }}\right)-\sum_{i=1}^{m} \sum_{h=1}^{n} p_{h} C_{i t}^{+} D_{i h t}^{+} \\
& \text {s.t. } \quad \sum_{i=1}^{m}\left(\Delta T_{i t} y_{i t o p t}-D_{i h t}{ }^{+}\right) \leq q_{h}{ }^{-}-\sum_{i=1}^{m} T_{i t}^{-}, \quad \forall h, t \\
& D_{\text {iht }}^{+}-\Delta T_{\text {it }} y_{\text {itopt }} \leq T_{i t}^{-}, \quad \forall i, h, t \\
& D_{\text {iht }}^{+} \geq D_{\text {ihtopt }}^{-}, \quad \forall i, h, t
\end{aligned}
$$

The sub-models (9) and (11) are deterministic linear stochastic programming problems. The solutions for model (6) under the optimized targets of allowable water allocation as follows:

$$
\begin{gathered}
f_{\text {topt }}^{ \pm}=\left[f_{\text {topt }}^{-}, f_{\text {topt }}^{+}\right] \\
\mathrm{D}_{\text {ihtopt }}^{ \pm}=\left[D_{\text {ihtopt }}^{-}, D_{\text {ihtopt }}^{+}\right], \quad \forall i, h, t
\end{gathered}
$$

where $f_{\text {opt }}^{+}$and $D_{\text {ihopt }}^{-}$are solutions of sub-model (9), and $f_{\text {opt }}^{-}$and $D_{\text {ihopt }}^{+}$are solutions of (11). Therefore, the optimal scheme of water allocation is:

$$
A_{\text {ihtopt }}^{ \pm}=T_{\text {itopt }}^{ \pm}-D_{\text {ihtopt }}^{ \pm}, \quad \forall i, h, t
$$

\section{Case Study}

\subsection{Overview of the Study System}

The following study case will be used to illustrate reliability and applicability within the TISP method. Dalian $\left(38^{\circ} 43^{\prime} \sim 40^{\circ} 10^{\prime} N, 120^{\circ} 58^{\prime} \sim 123^{\circ} 31^{\prime} \mathrm{E}\right)$, located at the southernmost tip of the Liaodong Peninsula, is an important port city of Liaoning. Associated with a warm temperate continental monsoon climate with a maritime feature, Dalian's annual average temperature is $10.5^{\circ} \mathrm{C}$ and annual average precipitation is from 600 to $800 \mathrm{~mm}$. The amount of water per capita in Dalian which is less than $1 / 4$ that of domestic is $493 \mathrm{~m}^{3}$. It is one of the cities that suffer severe water shortage problems in China. There are two major river systems in Dalian including Yellow sea basin and Bohai sea basin. Among them, Biliu River which is the largest river in Dalian city is a water source of urban inter-basin diversion. Currently the level of the exploitation and utilization of the water resources within six major rivers is very high.

In this case study, an amount of information regarding water resource demands and economic data should be identified firstly, then input to the water resource allocation model. However, parameter uncertainties exist in the system. Thus it is better to be expressed as interval parameters. Table 1 shows the related water availability and economic date of Dalian. Table 2 presents the probability distributions of the annual inflows. Let $\mathrm{i}=1,2,3$ for municipal, agricultural and industrial sector respectively. Let $\mathrm{h}=1$ for "streamflow level is low", $\mathrm{h}=2$ for "streamflow level is low-medium", $\mathrm{h}=3$ for "streamflow level is medium", $\mathrm{h}=4$ for "streamflow level is medium-high" and $\mathrm{h}=5$ for 
"streamflow level is high". The planning time horizon is 15 years. It is further divided to three different planning year, which $t=1,2,3$ is 2015, 2020, 2030 respectively. Note that, the data are estimated in terms of the Dalian sustainable development planning and statistical yearbooks.

Table 1. Water Resources and the Related Economic Data

\begin{tabular}{cccccc}
\hline $\begin{array}{c}\text { Planning } \\
\text { period }\end{array}$ & User & $\begin{array}{c}\text { Water allocation } \\
\text { target }\left(T_{i t}^{ \pm}\right) / 10^{4} \mathrm{~m}^{3}\end{array}$ & $\begin{array}{c}\text { Maximum } \\
\text { allowable } \\
\text { allocation } \\
\left(T_{i t m a x}^{ \pm}\right)\end{array}$ & $\begin{array}{c}\text { Net benefit when } \\
\text { water demand is } \\
\text { satisfied }\left(N B_{i t}^{ \pm}\right) \\
/ \text {Yuan } / \mathrm{m}^{3}\end{array}$ & $\begin{array}{c}\text { Recourse cost } \\
\text { when water is not } \\
\text { delivered }\left(C_{i \bar{t}}^{ \pm}\right) \\
/ \text {Yuan } / \mathrm{m}^{3}\end{array}$ \\
\hline \multirow{2}{*}{$\mathrm{t}=1$} & $\mathrm{i}=1$ & {$[28106,29456]$} & 3 & {$[3.5,4.0]$} & {$[4.2,4.8]$} \\
& $\mathrm{i}=2$ & {$[47905,54388]$} & 6 & {$[0.4,0.5]$} & {$[0.5,0.6]$} \\
& $\mathrm{i}=3$ & {$[38780,48404]$} & 5 & {$[588,625]$} & {$[705.6,750]$} \\
$\mathrm{t}=2$ & $\mathrm{i}=1$ & {$[36651,38283]$} & 4 & {$[5.0,5.5]$} & {$[7,7.7]$} \\
& $\mathrm{i}=2$ & {$[47664,55129]$} & 6 & {$[0.5,0.7]$} & {$[0.7,1]$} \\
& $\mathrm{i}=3$ & {$[51554,64118]$} & 7 & {$[625,666.7]$} & {$[875,933.4]$} \\
$\mathrm{t}=3$ & $\mathrm{i}=1$ & {$[51001,52995]$} & 6 & {$[6.5,7]$} & {$[10.4,11.2]$} \\
& $\mathrm{i}=2$ & {$[47084,58288]$} & 6 & {$[0.7,0.9]$} & {$[1.1,1.4]$} \\
& $\mathrm{i}=3$ & {$[89385,109155]$} & 11 & {$[666.7,706.7]$} & {$[1066.7,1130.7]$} \\
\hline
\end{tabular}

Table 2. Description of Streamflow Distribution

\begin{tabular}{ccc}
\hline & Seasonal flow $\left(q_{h}^{ \pm}\right) / 10^{4} \mathrm{~m}^{3}$ & Probability $\left(p_{h}\right)$ \\
\hline Low $(\mathrm{h}=1)$ & {$[84715,104922]$} & $5 \%$ \\
Low-medium $(\mathrm{h}=2)$ & {$[104922,197000]$} & $20 \%$ \\
Medium $(\mathrm{h}=3)$ & {$[197000,283910]$} & $25 \%$ \\
Medium-high $(\mathrm{h}=4)$ & {$[283910,425865]$} & $30 \%$ \\
High $(\mathrm{h}=5)$ & {$[425865,505237]$} & $20 \%$ \\
\hline
\end{tabular}

\subsection{Result Analysis}

The constrained optimization problems would be solved by the Lingo solver with the modeling language. Table 3 shows the optimal allowable water-allocation targets for each water user with the ITSP model. It is indicated that all of the optimal targets would reach the upper bounds which are presented in Table 1. In detail, when the water availability is low $(\mathrm{h}=1)$, water deficit for each user with different planning year is $D_{211 \text { opt }}^{ \pm}=[0,17686]$, $D_{212 o p t}^{ \pm}=[52608,55129], D_{113 o p t}^{ \pm}=52995, D_{213 o p t}^{ \pm}=58288, D_{313 o p t}^{ \pm}=[4233,24440]$ respectively, while that for the others is zero. It can be found that agricultural sector would be faced with the threat of water deficit firstly (Figure 1). The industrial sector is guaranteed because of its considerable benefit and high penalty. Moreover, as the development of socioeconomic promotes water demands for each user, the water shortage would increase from 2015 to 2030 . For instance, water shortage for users 1 and 2 are equal to their water-allocation targets in 2030, whereas user 3's water deficit is less than water demand. Therefore, the amount of water allocation $\left(A_{111 \text { opt }}^{ \pm}, A_{311 \text { opt }}^{ \pm}, A_{312 o p t}^{ \pm}\right)$for municipality and industry equal the total available water (Figure 2). When water availability is $[104922,197000] 10^{4} \mathrm{~m}^{3}$, water demand of municipality and industry should be satisfied firstly. From table $3, A_{221 o p t}^{ \pm}=[27062,54388], A_{222 o p t}^{ \pm}=[2521,55129]$, $A_{123 \text { opt }}^{ \pm}=[0,52995], A_{223 o p t}^{ \pm}=[0,34850], A_{323 \text { opt }}^{ \pm}=[104922,109155]$, while others have reached their upper bounds of water allocation. This implies that water demand of municipality and industry would be satisfied, and then the excess water could be assigned 
to agricultural sector in 2020. Due to insufficient water and increasing demand in 2030, three users would be faced with different degrees of water shortage. When water availability reaches $[197000,283910] 10^{4} \mathrm{~m}^{3}$, most of water demand could be satisfied except for user 2 in 2030. Under this circumstance, the priority of water allocation belongs to municipal, agricultural and then agricultural sector if applicable.

The net system benefits under optimized allowable water-allocation targets in three different planning year (Figure 3) are obtained. Extremely high net system benefits will be acquired under these water-allocation targets. The uncertainties result in wide intervals between the lower and upper bound of $f_{\text {topt }}^{ \pm}$. The results of $f_{\text {topt }}^{-}$and $f_{\text {topt }}^{+}$offer two extreme values of the net system benefit in the different planning year. Since the parameters and variables fluctuate between upper and lower bounds, the net system benefits would change between $f_{\text {topt }}^{-}$and $f_{\text {topt }}^{+}$correspondingly. The solutions when all $T_{i t o p t}^{ \pm}$come to the upper bounds of $T_{i t}^{ \pm}$represent that the authority is optimistic about water availability. In addition, both of the net system benefit and recourse cost will increase from 2015 to 2030 . For instance, the net system benefit would be [2858, 3040], [4027, 4299], and [7064, 7730] $10^{8}$ Yuan from 2015 to 2030 in Dalian, respectively. Thus, an optimistic policy would bring a higher net system benefit to each user meanwhile it may undertake a higher water shortage risk. Conversely, corresponding to conservative policy, a lower net system benefit will be obtained accompanied by a lower risk of water shortage as well as potential waste of water resource.

Table 3. Solution Under Optimized Water-Allocation Targets

\begin{tabular}{|c|c|c|c|c|}
\hline & & $\mathrm{i}=1$ & $i=2$ & $i=3$ \\
\hline \multicolumn{5}{|c|}{$\operatorname{Target}\left(T_{\text {itopt }}^{ \pm}\right) / 10^{4} \mathrm{~m}^{3}$} \\
\hline \multicolumn{2}{|c|}{$\mathrm{t}=1$} & 29456 & 54388 & 48404 \\
\hline \multicolumn{2}{|c|}{$\mathrm{t}=2$} & 38283 & 55129 & 64118 \\
\hline \multicolumn{2}{|c|}{$t=3$} & 52995 & 58288 & 109155 \\
\hline \multicolumn{5}{|c|}{$\operatorname{Deficits}\left(D_{\text {ihtopt }}^{ \pm}\right) / 10^{4} \mathrm{~m}^{3}$} \\
\hline \multirow{5}{*}{$t=1$} & $\mathrm{~h}=1$ & 0 & {$[27326,47533]$} & 0 \\
\hline & $h=2$ & 0 & {$[0,27326]$} & 0 \\
\hline & $h=3$ & 0 & 0 & 0 \\
\hline & $h=4$ & 0 & 0 & 0 \\
\hline & $h=5$ & 0 & 0 & 0 \\
\hline \multirow{5}{*}{$\mathrm{t}=2$} & $h=1$ & {$[0,17686]$} & {$[52608,55129]$} & 0 \\
\hline & $h=2$ & 0 & {$[0,52608]$} & 0 \\
\hline & $h=3$ & 0 & 0 & 0 \\
\hline & $h=4$ & 0 & 0 & 0 \\
\hline & $h=5$ & 0 & 0 & 0 \\
\hline \multirow{5}{*}{$t=3$} & $h=1$ & 52995 & 58288 & {$[4233,24440]$} \\
\hline & $h=2$ & {$[0,52995]$} & {$[23438,58288]$} & {$[0,4233]$} \\
\hline & $h=3$ & 0 & {$[0,23438]$} & 0 \\
\hline & $h=4$ & 0 & 0 & 0 \\
\hline & $\mathrm{h}=5$ & 0 & 0 & 0 \\
\hline \multicolumn{5}{|c|}{$\operatorname{Allocation}\left(A_{\text {ihtopt }}^{ \pm}\right) / 10^{4} \mathrm{~m}^{3}$} \\
\hline \multirow{5}{*}{$\mathrm{t}=1$} & $\mathrm{~h}=1$ & 29456 & {$[6855,27062]$} & 58404 \\
\hline & $h=2$ & 29456 & {$[27062,54388]$} & 58404 \\
\hline & $h=3$ & 29456 & 54388 & 58404 \\
\hline & $h=4$ & 29456 & 54388 & 58404 \\
\hline & $h=5$ & 29456 & 54388 & 58404 \\
\hline \multirow{2}{*}{$\mathrm{t}=2$} & $\mathrm{~h}=1$ & {$[20597,38283]$} & {$[0,2521]$} & 64118 \\
\hline & $\mathrm{h}=2$ & 38283 & {$[2521,55129]$} & 64118 \\
\hline
\end{tabular}




\begin{tabular}{ccccc} 
& $\mathrm{h}=3$ & 38283 & 55129 & 64118 \\
& $\mathrm{~h}=4$ & 38283 & 55129 & 64118 \\
$\mathrm{~h}=5$ & 38283 & 55129 & 64118 \\
$\mathrm{~h}=1$ & 0 & 0 & {$[84715,104922]$} \\
& $\mathrm{h}=2$ & {$[0,52995]$} & {$[0,34850]$} & {$[104922,109155]$} \\
$\mathrm{h}=3$ & 52995 & {$[34850,58288]$} & 109155 \\
$\mathrm{~h}=3$ & 52995 & 58288 & 109155 \\
& $\mathrm{~h}=4$ & 58288 & 109155 \\
\hline $\mathrm{h}=5$ & 52995 & & \\
\hline \multicolumn{2}{c}{ Objective $\left(f_{\text {topt }}^{ \pm}\right) / 10^{8}$ Yuan } & {$[2858,3040]$} \\
\multicolumn{2}{c}{$\mathrm{t}=1$} \\
$\mathrm{t}=2$ & & {$[4027,4299]$} \\
$\mathrm{t}=3$ & & {$[7064,7730]$} & \\
\hline
\end{tabular}

(a) $t=1$

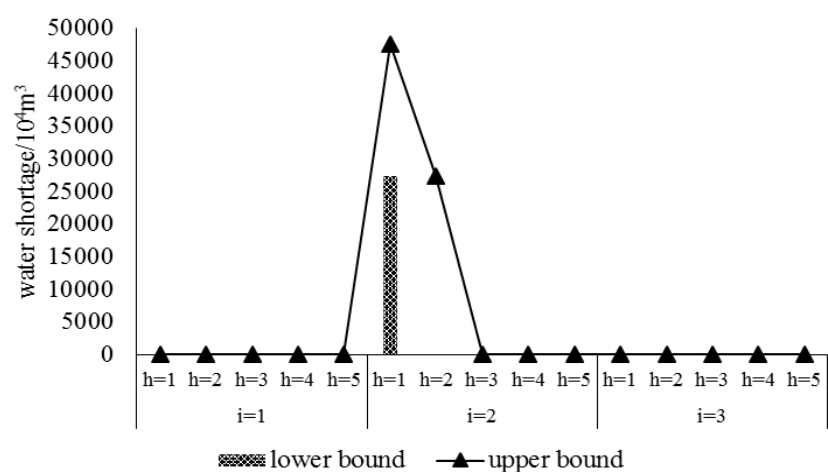

(b) $\mathrm{t}=2$

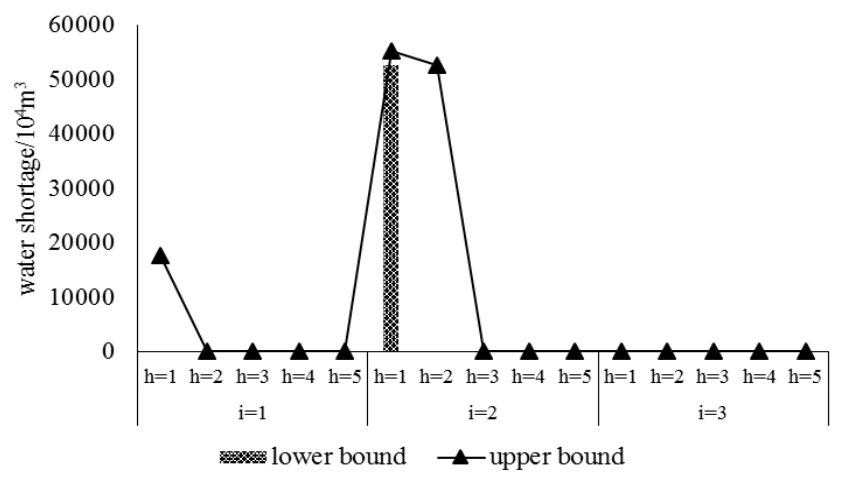

(c) $t=3$

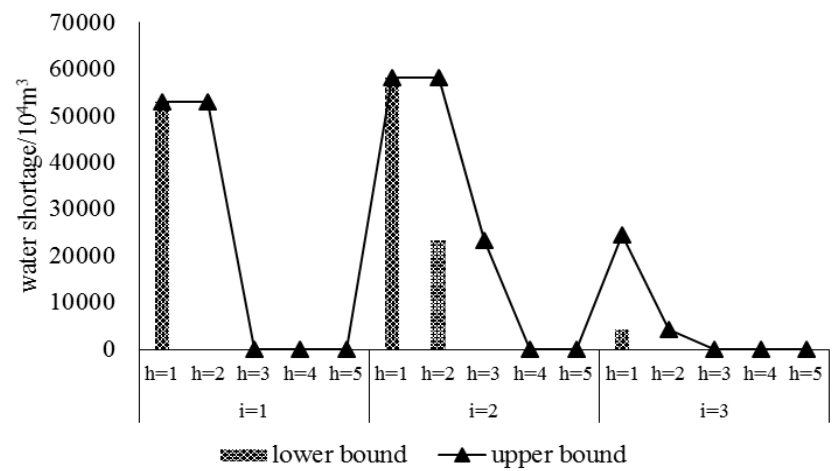

Figure 1. Water Allocation Deficits for Different Sector in the Three Different Planning Year 


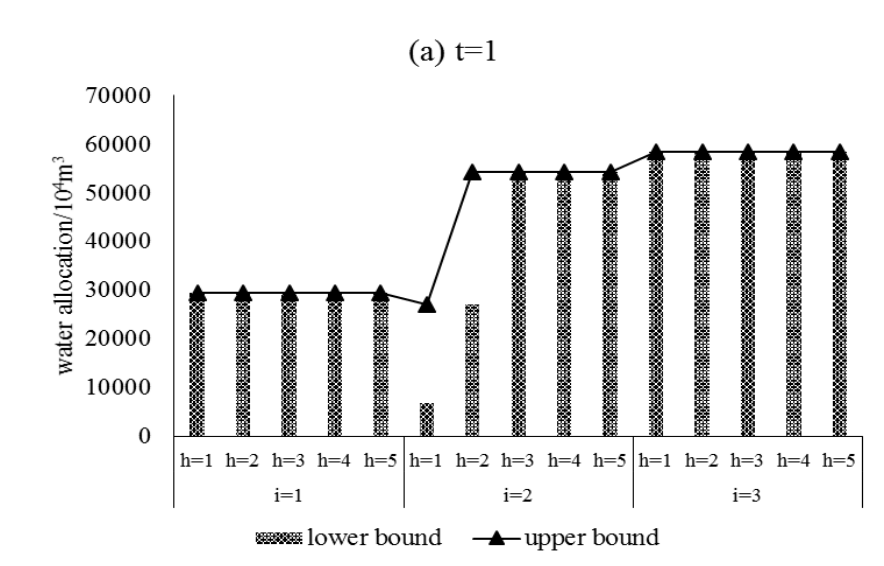

(b) $\mathrm{t}=2$

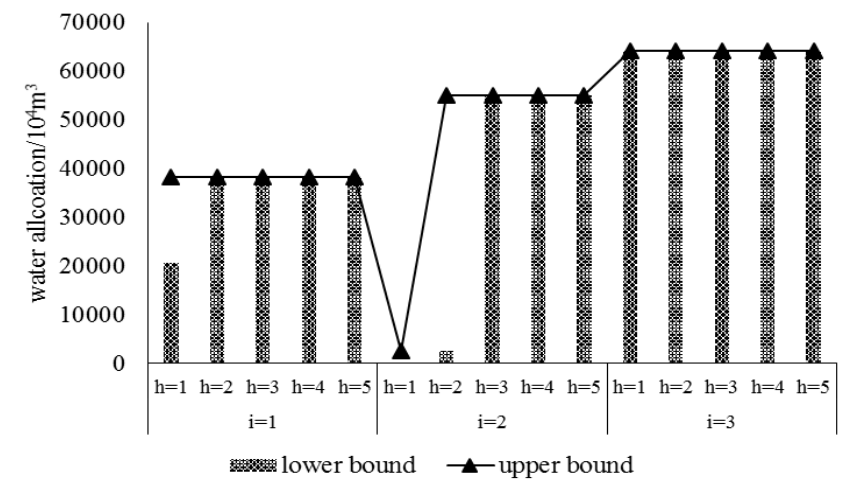

(c) $\mathrm{t}=3$

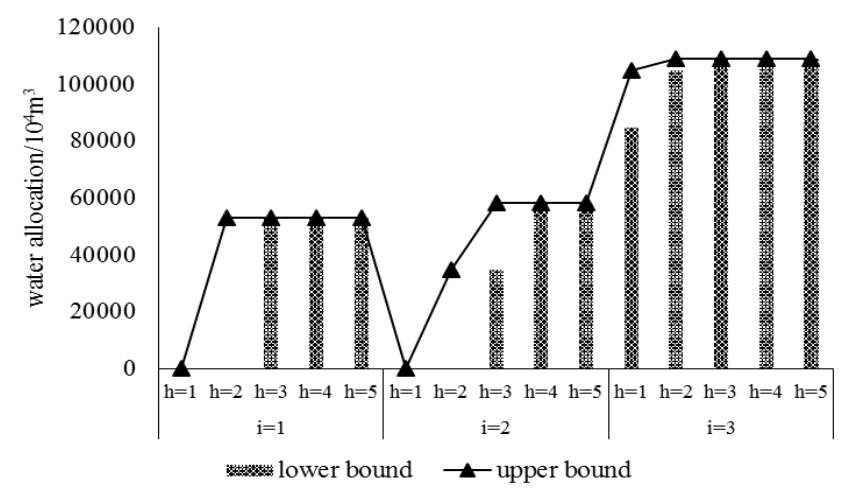

Figure 2. Optimal Water-Allocation Schemes for Different Sector in the Three Different Planning Year 


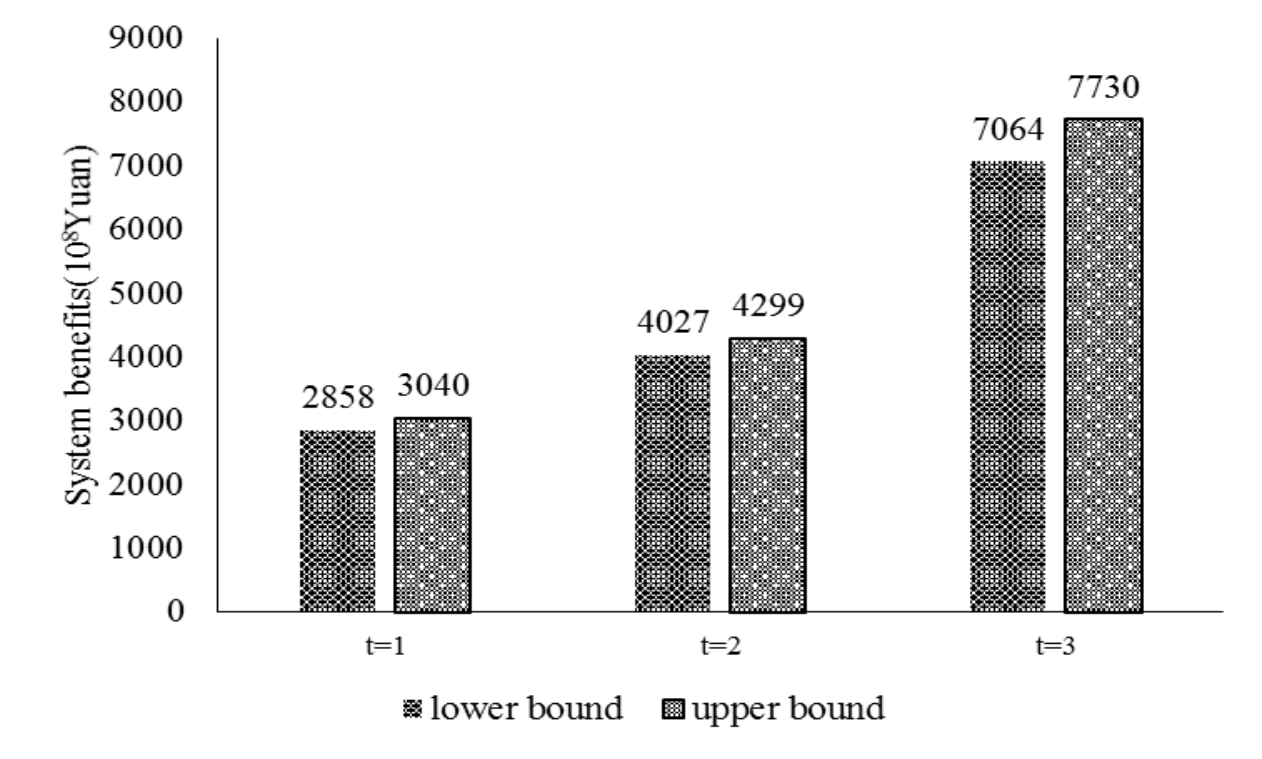

Figure 3. Net System Benefits in the Three Different Planning Year

\section{Conclusion}

In this paper, an inexact two-stage stochastic programming model was applied to sustainable utilization of water resource under uncertainty in Dalian city. The developed model, integrated the two-stage stochastic programing and inexact optimization, could deal with uncertain problems expressed as probability distributions and discrete intervals. The method provides an effective solution between conflicting system benefits and related recourse cost attributed to violating the pre-defined economic policy. In addition, the model that transformed into two deterministic optimized sub-models, which are corresponded to the upper and lower bound of the desired objective-function values, could obtain the interval solutions. By applied the model to a representative case of water allocation in the Dalian city in China, three water users, three different planning years were considered in the optimization model. The result indicated that different water allocation schemes and water deficits with varying net system benefits would be hold in response to different streamflow levels. However, these are still some deficiencies that limit the proposed model. In detail, the maximum net system benefit was the only factor to be considered in the objective function. The risk of model reliability and feasibility were not taken into account. The limitation could lead to unbalanced allocation pattern and reduce system stability. More developed methods, such as Monte Carlo inexact twostage stochastic model, fuzzy-incredibility constrained two-stage stochastic model and hybrid approaches should be incorporated to obtain improving applicability. What's more, only three water user sectors were considered in this study. This may not reflect the overall water demand situation of Dalian city. So the integration of such features into the model would be the future topic that deserve to be researched.

\section{Acknowledgment}

This work has been supported by the National Science Foundation for Innovative Research Group (No.51121003) and the Fundamental Research Funds for the Central Universities (2014KJJCB10). 


\section{References}

[1] Y.P. Cai, G.H. Huang, X. Wang, G.C. Li and Q. Tan, "An inexact programming approach for supporting ecologically sustainable water supply with the consideration of uncertain water demand by ecosystems", Stochastic Environmental Research and Risk Assessment, vol 25, no 5, (2011), pp. 721-735.

[2] UN-Water. The united nation world water development report; the United Nations Educational, Scientific and Cultural Organization, (2014).

[3] L. Li, X.M. Ding and K. Qi, "The application of fuzzy data mining in water resource management", Journal of Harbin University of Science and Technology, vol 11, no 6, (2006), pp. 99-101.

[4] J.R. Birge and F.V. Louveaux, "A multicut algorithm for two-stage stochastic linear programs", European Journal of Operational Research, vol 34, no 3, (1988), pp. 384-392.

[5] A. Loukas, N. Mylopoulos and L. Vasiliades, "A modeling system for the evaluation of water resources management strategies in thessaly, greece", Water Resources Management, vol 21, no 10, (2007), pp. 1673-1702.

[6] Y.L. Xie, G.H. Huang, W. Li, J.B. Li and Y.F. Li, "An inexact two-stage stochastic programming model for water resources management in nansihu lake basin, china", Journal of Environmental Management, vol 127, (2013), pp. 188-205.

[7] J. Liu, Y.P. Li, G.H. Huang and X.T. Zeng, "A dual-interval fixed-mix stochastic programming method for water resources management under uncertainty", Resources, Conservation and Recycling, vol 88, (2014), pp. 50-66.

[8] X.T. Zeng, Y.P. Li, W. Huang, X. Chen and A.M. Bao, "Two-stage credibility-constrained programming with hurwicz criterion (tcp-ch) for planning water resources management", Engineering Applications of Artificial Intelligence, vol 35, (2014), pp. 164-175.

[9] S. Wang and G.H. Huang, "A multi-level taguchi-factorial two-stage stochastic programming approach for characterization of parameter uncertainties and their interactions: An application to water resources management", European Journal of Operational Research, vol 240, no 2, (2015), pp. 572-581.

[10] D. Wang and B.J. Adams, "Optimization of real-time reservoir operations with markov decision processes", Water Resources Research, vol 22, no 3, (1986), pp. 345-352.

[11] N.C.P. Edirisinghe and W.T. Ziemba, "Bounds for two-stage stochastic programs with fixed recourse", Mathematics of Operations Research, vol 19, no 2, (1994), pp. 292-313.

[12] S.K. Barik, M.P. Biswal and D. Chakravarty, "Two-stage stochastic programming problems involving multi-choice parameters", Applied Mathematics and Computation, vol 240, (2014), pp. 109-114.

[13] H.W. Lu, G.H. Huang, G.M. Zeng, I. Maqsood and L. He, "An inexact two-stage fuzzy-stochastic programming model for water resources management", Water Resources Management, vol 22, no 8, (2008), pp. 991-1016

[14] Y.R. Fan, G.H. Huang, P. Guo and A.L. Yang, "Inexact two-stage stochastic partial programming: Application to water resources management under uncertainty", Stochastic Environmental Research and Risk Assessment, vol 26, no 2, (2012), pp. 281-293.

[15] Y. Zhou and Z.X. Hao, "Model for indeterminable temporal information representation based on porbability", Journal of Harbin University of Science and Technology, vol 13, no 2, (2008), pp. 15-18.

[16] G.H. Huang and D.P. Loucks, "An inexact two-stage stochastic programming model for water resources management under uncertainty", Civil Engineering and Environmental Systems, vol 17, no 2, (2000), pp. 95-118.

[17] I. Maqsood, G.H. Huang and G.M. Zeng, "An inexact two-stage mixed integer linear programming model for waste management under uncertainty", Civil Engineering and Environmental Systems, vol 21, no 3, (2004), pp. 187-206.

[18] Y.P. Li and G.H. Huang, "Two-stage planning for sustainable water-quality management under uncertainty", Journal of Environmental Management, vol 90, no 8, (2009), pp. 2402-2413.

[19] P. Guo, G.H. Huang, H. Zhu and X.L. Wang, "A two-stage programming approach for water resources management under randomness and fuzziness", Environmental Modelling \& Software, vol 25, no 12, (2010), pp. 1573-1581.

[20] B. Luo, I. Maqsood, Y. Y. Yin, G. H. Huang and S.J. Cohen, "Adaption to climate change through water trading under uncertainty - an inexact two-stage nonlinear programming approach ", Journal of Environmental Informatics, vol 2, no 2, (2003), pp. 58-68.

[21] Y.L. Xie and G.H. Huang, "Development of an inexact two-stage stochastic model with downside risk control for water quality management and decision analysis under uncertainty", Stochastic Environmental Research and Risk Assessment, vol 28, no 6, (2014), pp. 1555-1575.

[22] G.H. Huang, "A hybrid inexact-stochastic water management model", European Journal of Operational Research, vol 107, no 1, (1998), pp. 137-158.

[23] G.H. Huang, "Ipwm: An interval parameter water quality management model. Eng opt", Engineering Optimization, vol 26, no 2, (1996), pp. 79-103. 
International Journal of Smart Home

Vol. 10, No. 5 (2016) 